\title{
In Situ Imaging of Langmuir Films Using Environmental Scanning Electron Microscopy
}

\author{
A.F. Miller* and S.J. Cooper** \\ * Polymer and Colloid Group, Cavendish Laboratory, University of Cambridge, Cambridge, CB3 \\ OHE. \\ ** Department of Chemistry, University of Durham, Durham, DH1 3LE.
}

Environmental scanning electron microscopy (ESEM) has been applied to characterize the organization of spread films at the gas-water interface directly. This recent extension of conventional high vacuum SEM allows for high resolution electron imaging of insulated and hydrated samples maintained in their natural state. ESEM therefore has great potential for the study of structure and dynamics of liquid surfaces. We have applied this novel technique to thein-situ imaging of a nylon 6 6 polymer spread film as a function of surface concentration ${ }^{1}$. To our knowledge, this is the first insitu ESEM study of a spread film on a liquid surface. The technique offers significantly better resolution over other in-situ imaging techniques such as Brewster angle and optical microscopy, and enables dynamic studies upon spread films in their natural state to be carried out.

Nylon 66 polymer films were prepared by depositing typically $50 \mu$ of polymer solution $(0.5 \mathrm{mg}$ $\mathrm{ml}^{-1}$ where the solvent was phenol-toluene (1:3)) onto an ultrapure water surface. For ESEM experiments the system was contained in a circular brass stub with a diameter of $2 \mathrm{~cm}$ and a depth of $1 \mathrm{~cm}$. To ensure that the film organisation adopted in the ESEM sample chamber (where the subphase temperature is held constant at $275 \mathrm{~K}$ ) is representative of the polymer conformation at room temperature, surface pressure isotherms were obtained at the two temperatures, 275 and $298 \mathrm{~K}$. Comparing the isotherms obtained reveals that characteristic film behaviour is not dependent on temperature (within this experimental range) as there is minimal difference between surface pressure curves within the limits of reproducibility.

Monolayer structure was subsequently examined over a range of surface concentrations encompassing regions of interest from the surface pressure isotherm data. It was found that at all surface concentrations studied the individual nylon chains $(3.7 \AA$ wide) aggregate through hydrogen bonding to form microfibrils that align parallel to the water surface. At low surface concentrations $\left(0.3-0.8 \mathrm{mg} \mathrm{m}^{-2}\right)$ corresponding to the expanded region of the surface pressure isotherm $(\pi \sim 0-2 \mathrm{mN}$ $\mathrm{m}^{-1}$ ), the polymer films formed were not continuous but instead dispersed in islands on the surface that moved randomly across the surface. A typical snapshot image (rapid scan speed) for such a cluster of polymer material is provided in figure 1.

As the surface coverage increased, the mobility of these islands reduced while they increased in size until the limiting surface coverage of $1.0 \mathrm{mg} \mathrm{m}^{-2}$, at a nominal $\pi \sim 3 \mathrm{mN} \mathrm{m}^{-1}$. At this concentration a continuous film was observed where the microfibrils were close-packed and $\sim 700 \mathrm{~nm}$ in length with a width of $\sim 20 \mathrm{~nm}$. In this surface concentration region the degree of two-dimensional ordering is high and from figure 3 it is evident that the individual fibrils align parallel with each other and to the water surface most probably due to hydrogen bonding between the amide groups. High resolution images revealed that the distance between the microfibrils decreased with further concentration increases. 
The morphologies observed are qualitatively comparable to optical micrographs obtained by collapsing nylon 66 films under high surface pressure to form thicker films ${ }^{2}$, and to scanning force micrographs obtained from films deposited upon mica substrates ${ }^{3}$. The findings are also supported by external reflection FTIR studies ${ }^{1}$. The experimental procedure and conditions for this novel experiment will be presented and the results obtained discussed in relation to those from conventional methods.

References

[1] A.F. Miller and S.J. Cooper, Langmuir 18 (4) (2002) 1310.

[2] L.F. Wang et.al., Mater. Chem. Phys. 40 (1995) 197.

[2] R. Popovitz-Biro et.al., Acta. Polym. 49 (1998) 626.

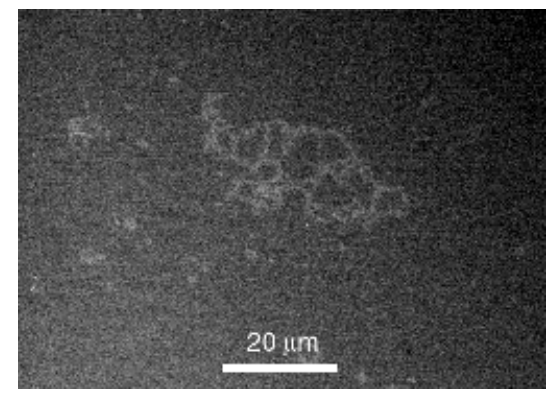

FIG. 1. ESEM micrograph of an island of polymer moving across the water surface at surface concentration is $0.5 \mathrm{mg} \mathrm{m}^{-2}$. Note the quality of the image is low due to the rapid movement across the water surface(Beam energy was $10 \mathrm{keV}$ and the chamber pressure and sample temperature are 4.2 Torr and $275 \mathrm{~K}$ respectively).
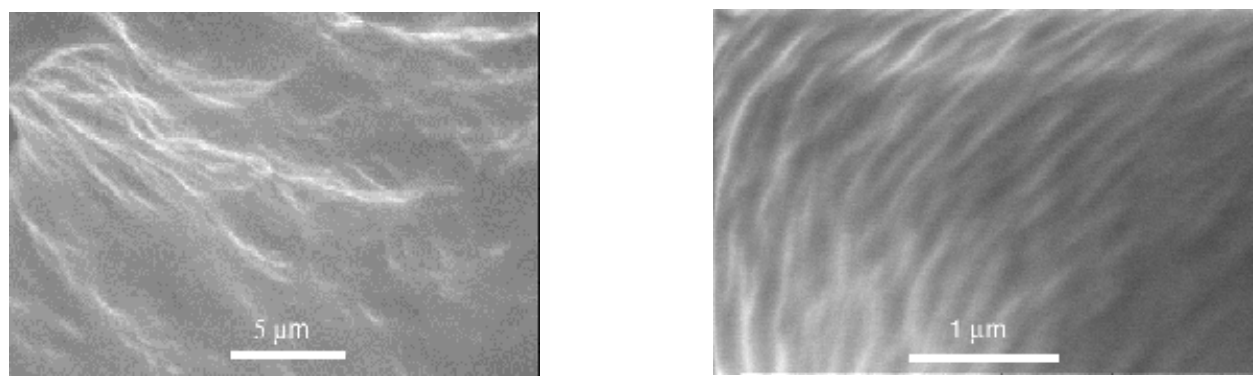

FIG. 2. ESEM micrographs of polymer film at a surface concentration of $1.0 \mathrm{mg} \mathrm{m}^{-2}$ (Beam energy was $10 \mathrm{keV}$ and the chamber pressure and sample temperature are 4.2 Torr and $275 \mathrm{~K}$ respectively). 